\title{
Arztinformationssysteme
}

\section{Arznei ohne Zusatznutzen - aber wichtig für die Versorgung}

\author{
Der gemeinsame Bundesausschuss arbeitet darauf hin, dass die von ihm herausgegebenen frühen \\ Nutzenbewertungen die verordnenden Ärzte stärker als bisher erreichen. Bei der Konzeption eines solchen \\ Arztinformationssystems will der Bundesausschuss besonderen Wert auf die Konstellationen legen, in denen \\ Arzneien kein Zusatznutzen anerkannt wurde. Denn auch sie seien für die Versorgung von Bedeutung.
}

$\mathrm{D}$ ie Originaldokumente des Gemeinsamen Bundesausschusses (GBA) zur frühen Nutzenbewertung - Beschlusstexte und tragende Gründe - erreichen bislang meist nur Experten, Insider und Methodiker, aber kaum die verordnenden Ärzte. Insofern, so bedauert der Leiter der GBA-Abteilung Arzneimittel, Thomas Müller, haben die Bewertungsergebnisse wenig Einfluss auf die Versorgung. Das solle sich mit dem in die Praxissoftware eingebauten Arztinformationssystem ändern, kündigte Müller bei einem von Boehringer Ingelheim organisierten Symposion in Berlin an.

Dabei will der vom Gesetzgeber beauftragte Bundesausschuss auch die Kompetenz von Kommunikationswissenschaftlern nutzen, um für Ärzte und Patienten zugängliche Informationsformate zu schaffen.

Inhaltlich werde der Bundesausschuss besonderes Augenmerk auf Informationen über jene Arzneimittel und Patientenpopulationen richten, für die der G-BA keinen Zusatznutzen als nachgewiesen angesehen habe. Denn das Urteil über den Innovationsgrad auf der Systemebene könne nicht eins-zu-eins auf die Versorgungsebene im Einzelfall übertragen werden, konzedierte Müller. In einer Konstellation ohne Zusatznutzen sei das betreffende Arzneimittel zwar nicht im Regelfall einsetzbar, wenn der Erstattungsbetrag über dem Preis der Vergleichstherapie liege - gleichwohl müsse ein solches Arzneimittel als Therapieoption oder -alternative zu Verfü- gung stehen. Dies setze eine verantwortliche Entscheidung des Arztes - auch unter Wirtschaftlichkeitsaspekten - voraus. Vor allem in den tragenden Gründen könne Ärzten eine Hilfestellung gegeben werden. Darüber hinaus müssten den Ärzten die Bedingungen für eine qualitätsgesicherte Anwendung bekannt sein: die geforderte ärztliche Qualifikation, Diagnostik und Dokumentation.

Wie ein solches Arztinformationssystem aussehen könnte, macht die Deutsche Gesellschaft für Hämatologie und Medizinische Onkologie (DGHO) mit einer "Onkopedia“ vor, die die Nutzenbewertung in Kurzfassung auf ihrer Website abbildet und durch Informationen wie aktuelle Leitlinien und Studien ergänzt. Ein solches Informationssystem sollte den komplexen Entscheidungsprozess des Arztes unter Einbeziehung aller Therapieoptionen und Diagnostik einbeziehen, fordert Prof. Bernhard Wörmann von der DGHO.

Beispiele verdeutlichen, wie rasch Entscheidungen des G-BA überholt sein können: Osimertinib, bewertet im September 2016 auf magerer Studienbasis mit dem Ergebnis „kein Zusatznutzen“. Die Folge: ein Opt out des Herstellers. Im Dezember 2016: Vorlage einer PhaseIII-Studie mit guten Ergebnissen - derzeit wird Osimertinib aus Italien importiert und mit Billigung des MDK auch von den Kassen bezahlt.

Jüngstes Beispiel: die Negativbewertung von Crizotinib bei Patienten mit ROS1-positivem, nichtkleinzelligem Lungenkarzinom (NSCLC), an dem 300

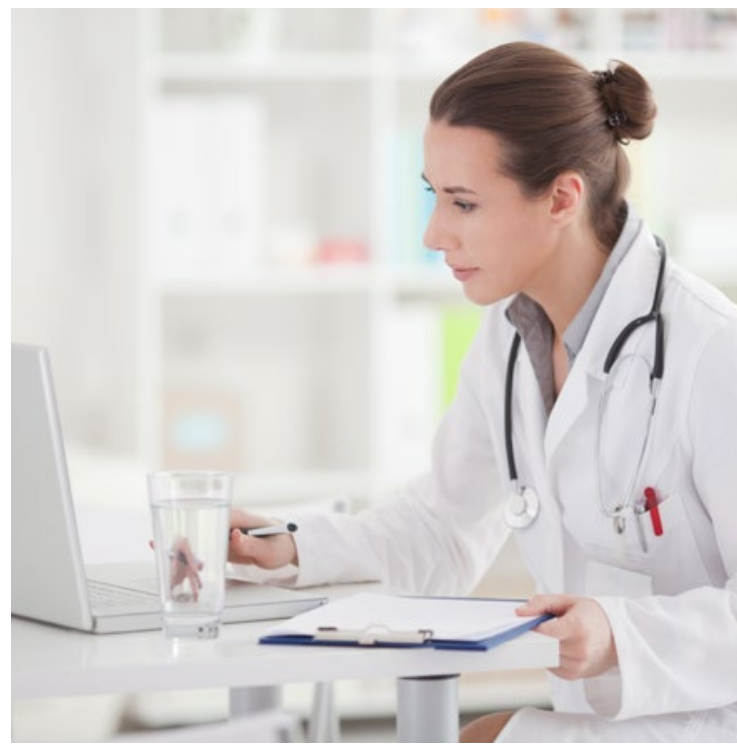

Ein Arztinformationssystem soll dabei helfen, dass Ärzte die frühe Nutzenbewertung in ihren Alltag integrieren.

bis 400 Patienten pro Jahr erkranken. Am 16. März hatte der Bundesausschuss vor dem Hintergrund einer nicht befriedigenden Studienlage entschieden: „Zusatznutzen nicht belegt" - aber zugleich konzediert: „Für nicht vorbehandelte Patienten mit ROS1-positiven NSCLC kann eine Behandlung (...) mit Crizotinib eine relevante Therapieoption sein." Das, so Prof. Carsten Brokemeyer von der DGHO, zeige das Dilemma des G-BA, der Verständnis für den verordnenden Arzt habe, zugleich aber gefangen sei im Korsett der eigenen Regeln.

Helmut Laschet 